\title{
Análises espaciais de doenças diarreicas e sua relação com o monitoramento ambiental
}

\author{
Spatial analysis of diarrheal diseases and its \\ relationship with the environmental monitoring
}

Francisco Rossarolla Forgiarini'* $\odot$, Robson Leo Pachaly' ${ }^{1}$, Jean Favaretto' ${ }^{\oplus}$

\begin{abstract}
RESUMO
A urbanização no Brasil, em geral, causa diversos problemas ambientais e sociais, destacando-se os de saúde pública. O presente estudo analisou o uso de ferramentas de geoprocessamento que podem ajudar a compreender a relação entre a qualidade da água em rios e a incidência de doenças diarreicas agudas. Em um município brasileiro, realizou-se o monitoramento ambiental em pontos de um rio urbano, buscou-se informações quanto às doenças diarreicas e utilizaram-se as ferramentas de geoprocessamento: análises de distribuição espacial com a ferramenta distância mais próxima e a estimativa de densidade kernel; e análises de aglomerados com a média do vizinho mais próximo e a função $K$ de Ripley. Entre os resultados, destaca-se: o ponto com a pior qualidade da água agrupou a maioria dos casos de doenças diarreicas, corroborando a associação clássica que relaciona este resultado às piores condições de vida; entre as ferramentas utilizadas, a estimativa de densidade kernel possibilitou a visualização espacial da aglomeração dos casos de diarreia; e a função $K$ de Ripley comprovou estatisticamente essa aglomeração. As informações do monitoramento ambiental dos rios contribuem para identificar as possíveis causas das doenças diarreicas. As análises espaciais realizadas podem ser replicadas em outros municípios com o objetivo de identificar áreas de risco às doenças diarreicas que possibilitem ações de prevenção.
\end{abstract}

Palavras-chave: distribuição espacial; análise de aglomerados; kernel; qualidade da água; função de Ripley.

\begin{abstract}
Overall, urbanization in Brazil causes many environmental and social problems, among which we highlight the public health impacts. This study examined the use of geoprocessing tools that can help understand the relationship between the quality of water in rivers and the incidence of acute diarrheal diseases. In a Brazilian municipality, we carried out environmental monitoring in points of a river, then sought information as to waterborne diseases, and used the following geoprocessing tools: spatial distribution analysis with distance nearest tool and kernel density estimation; and cluster analysis with the average nearest neighbour and the K Ripley function. Among the results are the following: the point with the worst water quality groups most of the cases of diarrheal diseases, corroborating the classical association that links this result to the worst living conditions; among the tools used, the kernel density estimation enabled spatial visualization of clustering of cases of diarrhea; and Ripley's $K$ function statistically demonstrated this agglomeration. The information derived from the environmental monitoring of rivers contributes to identify the possible causes of diarrheal diseases. The spatial analyses conducted can be replicated in other municipalities, in order to identify risk areas to diarrheal diseases and to allow preventive actions.
\end{abstract}

Keywords: spatial distribution; cluster analysis; kernel; water quality Ripley function.

\section{INTRODUÇÃO}

No Brasil, a péssima qualidade dos recursos hídricos é um dos principais problemas ambientais (VIANA; FREITAS; GIATTI, 2016). Os mananciais hídricos próximos às áreas urbanas merecem atenção especial em virtude da poluição difusa e de lançamentos de águas residuais de forma concentrada, prejudicando os múltiplos usos da água. Além disso, a poluição provoca a proliferação de microrganismos patogênicos que causam doenças de veiculação hídrica. Essas doenças são causadas principalmente por microrganismos oriundos do sistema digestório de animais homeotérmicos, transmitidos pela rota feco-oral (AMARAL et al., 2003; VIANA; FREITAS; GIATTI, 2016).

Atualmente, ferramentas de geoprocessamento estão sendo usadas para auxiliar no estudo de doenças de veiculação hídrica (TORRES et al., 2013). Nesse contexto, a vigilância em saúde tem se beneficiado dessas metodologias, que possibilitam a avaliação de hipóteses de riscos, que incorporam, entre outras, as variáveis ambientais e

$\square$ 
socioeconômicas (RIBEIRO, 2014). Exemplos podem ser citados para o controle da dengue (SIQUEIRA-JUNIOR et al., 2008), da leptospirose (VILAÇA et al., 2009), da hanseníase (CARVALHO; SOUZA-SANTOS, 2005) e de doenças diarreicas (QUEIROZ; HELLER; SILVA, 2009; TORRES et al., 2013).

Merecem destaque as análises históricas de casos de doenças ocorridas na Inglaterra e realizadas por Diggle e Chetwynd (1991) e Gatrell et al. (1996), por meio do uso do geoprocessamento. No primeiro estudo, os autores investigaram casos de leucemia infantil e linfoma, e constataram que as ocorrências dos casos teriam certo padrão de aglomeração espacial em uma distância de $500 \mathrm{~m}$. No segundo, foram verificados os padrões nas ocorrências de câncer infantil, linfoma de Burkitt, cancro da laringe e asma infantil, em que se averiguou que a escala da informação influenciou nos resultados, quando se analisaram as ocorrências ao nível de condado e de bairro separadamente.

As análises de distribuição espacial fornecem estatísticas que descrevem a distribuição espacial global, ou efeitos de primeira ordem. Segundo Druck et al. (2004), os efeitos de primeira ordem são aqueles que servem para estimar a intensidade pontual de um processo em uma determinada região (número de eventos por unidade de área). Tais efeitos são considerados globais ou de grande escala e correspondem a variações no valor médio do processo.

Por sua vez, a análise de aglomerados ou efeitos de segunda ordem representa a dependência espacial das ocorrências estudadas, e são denominados locais ou de pequena escala (CÂMARA; CARVALHO, 2004). Esta análise serve para verificar se os eventos observados apresentam algum tipo de padrão sistemático, ao invés de estarem distribuídos aleatoriamente.

Alguns dos desafios associados aos estudos de epidemiologia espacial foram abordados por Beale et al. (2008). Estes autores constataram que, mesmo com o aumento da disponibilidade de dados, métodos e tecnologias, os sistemas de monitoramento são recentes e deficientes, pois seus dados nem sempre possuem as informações necessárias para alimentar os modelos da estatística geoespacial. Assim, estudos que busquem conciliar as técnicas disponíveis com os atuais sistemas de monitoramento têm suma importância, não só com caráter informativo, mas também no intuito de embasar novas pesquisas.

Entre as doenças de veiculação hídrica, as diarreicas possuem muitas informações disponíveis no Brasil (QUEIROZ; HELLER; SILVA, 2009). A vigilância epidemiológica de casos individuais de doenças diarreicas preconizada pelo Ministério da Saúde foi criada em 1994, sendo chamada de Sistema de Monitorização das Doenças Diarreicas Agudas (MDDA). Esse sistema tem como objetivos monitorar a incidência das diarreias nos municípios brasileiros, visando detectar precocemente surtos da doença; investigar suas causas; e manter atividades contínuas de educação em saúde com o propósito de diminuir sua incidência e letalidade (QUEIROZ; HELLER; SILVA, 2009).
Queiroz, Heller e Silva (2009) salientam que a qualidade e/ou quantidade de água são fatores determinantes, ou ao menos fatores de risco, para as doenças diarreicas agudas (DDA). Os autores enfatizam ainda a importância e os benefícios que as intervenções ambientais proporcionam para a minimização das doenças diarreicas, e como os investimentos em saneamento têm consequências positivas na saúde pública.

Nesse contexto, o nosso objetivo foi analisar ferramentas de geoprocessamento que podem ajudar a compreender a relação entre a qualidade da água em um rio urbano e a incidência de DDA.

\section{METODOLOGIA}

O estudo foi desenvolvido no município de Silveira Martins, no interior do estado do Rio Grande do Sul. A metodologia do trabalho envolveu o desenvolvimento de três etapas.

\section{Análises qualitativas da água}

Foram realizadas 12 campanhas de qualidade de água com frequência mensal entre os anos de 2011 e 2012, em 3 pontos de monitoramento em um rio urbano. $\mathrm{O}$ ponto 1 (P1) está a montante do reservatório da Companhia Riograndense de Saneamento (CORSAN) que abastece o município, em uma área rural. $\mathrm{O}$ ponto $2(\mathrm{P} 2)$, denominado Vila Brasília, localiza-se a jusante da área urbana de Silveira Martins. O ponto 3 (P3), denominado Monumento, localiza-se em uma área de floresta nativa a cerca de 1,5 km da área urbana. As bacias 1, 2 e 3, formadas a partir dos pontos de monitoramento, possuem 0,72, 3,96 e $5,29 \mathrm{~km}^{2}$ de área, respectivamente. Um mapa com a localização dos pontos de monitoramento e das suas bacias é exibido na Figura 1.

As análises qualitativas da água foram: demanda bioquímica de oxigênio (DBO), oxigênio dissolvido (OD), temperatura, coliformes totais e termotolerantes (gênero Escherichia) e demais gêneros de

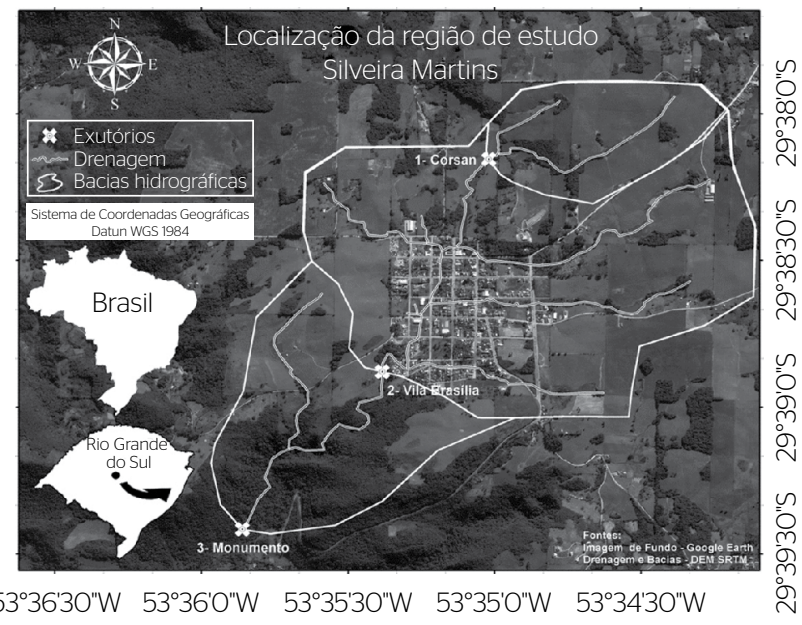

Figura 1 - Localização da região do estudo. 
bactérias presentes. A DBO foi determinada pelo método modificado de Winkler; o OD e a temperatura, por meio do oxímetro da marca DIGIMED, modelo DM4; os coliformes totais e termotolerantes foram quantificados pela técnica de tubos múltiplos (APHA, 2012). Os demais gêneros de bactérias foram determinados utilizando placas contendo o meio de cultura ágar padrão para a contagem (PCA).

\section{Serviço de esgotamento sanitário e doenças diarreicas no município}

As informações referentes aos serviços de esgotamento sanitário foram obtidas a partir do trabalho de Moraes, Santos e Forgiarini (2013). Quanto às doenças diarreicas, a busca de informações foi realizada na Secretaria de Vigilância Sanitária e no hospital do município, que fazem parte do Sistema MDDA. Foi organizado um Sistema de Informação Geográfica (SIG) usando o programa ArcGIS, e os casos de diarreia foram localizados no espaço, sendo analisada a distribuição espacial de acordo com as zonas do Plano Diretor do município. O Plano Diretor do município de Silveira Martins contempla oito zonas: zona central, zona mista, zona residencial 1, zona residencial 2, zona universitária, parque municipal, Zona Especial de Interesse Social e áreas de preservação permanente (APPs).

\section{Análises espaciais}

As análises espaciais compreenderam o uso de ferramentas de geoprocessamento de modo a evidenciar a relação entre os parâmetros de qualidade da água e a ocorrência dos casos de diarreia. Foram realizadas análises de distribuição espacial e de aglomerados com os programas QGIS e ArcGIS, e posteriormente comparadas com as espacializações das informações qualitativas da água.

\section{Análises de distribuição espacial}

\section{Análise de distância mais próxima}

A ferramenta de análise espacial de distância mais próxima foi utilizada para identificar a quais pontos de monitoramento os casos de diarreia estão mais próximos, ou seja, podem possuir uma possível associação espacial. Para a realização dessa análise foi utilizada a ferramenta Distance to Nearest Hub, do software QGIS versão 10.8.6.

A ferramenta realiza iterações entre diferentes feições com o intuito de encontrar a distância mais próxima entre elas. Neste estudo, a ferramenta foi utilizada para encontrar a menor distância da localização geoespacial de cada caso de diarreia com o ponto de monitoramento mais próximo.

\section{Estimativa de densidade kernel}

De acordo com Câmara et al. (2004), essa função realiza um cálculo de todos os pontos contidos em determinada região, ponderando-os pela distância de cada ponto a sua localização de interesse. Foi empregado o programa ArcGIS, que utiliza em seu estimador kernel a função quadrática apresentada por Silverman (1986), conforme Equação 1:

$f(x, y)=\frac{1}{n h^{2}} \sum_{i=1}^{n} k\left(\frac{d i}{h}\right)$

Em que:

$f(x, y)=$ valor da densidade no ponto $(x, y)$;

$\mathrm{n}$ = número de observações;

$\mathrm{h}=$ raio de pesquisa;

di = distância entre a observação i e o ponto $(x, y)$;

$\mathrm{k}=$ função densidade (kernel);

A estimativa de densidade de kernel (EDK) permite ao analista configurar três parâmetros (ESRI, 2015). O primeiro a ser atribuído é o tamanho da célula (TC), que significa o tamanho do pixel do arquivo Raster de saída que vai ser criado. O segundo parâmetro é o raio de pesquisa (RP), que cria a suavização da superfície, isto é, o valor da superfície será maior quanto mais próximo um ponto for do outro, sendo o oposto verdadeiro, e nula quando a distância entre os pontos for igual a do RP. E o último parâmetro permite ao analista a escolha do método para o cálculo das distâncias entre as feições, utilizando-se de distâncias planares ou geodésicas.

Para comparar quais parâmetros serão os mais indicados para o caso em estudo, usaremos a técnica proposta por Chainey, Tompson e Uhlig (2008), chamada de prediction accuracy index (PAI). Essa técnica divide a taxa de acerto, que é a porcentagem de novos casos que ocorrerão junto às áreas quentes, pela porcentagem de áreas quentes encontradas na extensão da área de estudo. Dessa forma, tem-se a Equação 2.

$\frac{(\mathrm{n} / \mathrm{N}) \times 100}{(\mathrm{a} / \mathrm{A}) \times 100}=\frac{\text { Taxa de acerto }}{\text { Porcentagem de áreas }}=\mathrm{PAI}$

Em que:

$\mathrm{n}$ = número de casos que são previstos de ocorrer (áreas quentes);

$\mathrm{N}$ = número de casos na área de estudo;

$\mathrm{a}=$ área onde casos são previstos de ocorrer (áreas quentes);

A = área de estudo.

Segundo Chainey, Tompson e Uhlig (2008), quanto maior o valor de PAI, maior será o número de casos previstos dentro de menores áreas quentes, mostrando que quanto maior o valor de PAI mais representativo será a EDK.

\section{Análises de aglomerados}

\section{Média do vizinho mais próximo}

Empregou-se a média do vizinho mais próximo (VMP) para analisar se existe aglomeração dos casos de diarreia. Essa função mede a 
distância entre cada ponto e o seu vizinho mais próximo para, posteriormente, realizar a média de todas as distâncias (ESRI, 2015). Se a distância calculada entre os pontos é menor que a distância de uma distribuição hipotética aleatória, o conjunto de pontos segue uma tendência para aglomeração. O índice da VMP é determinado dividindo-se a distância média entre pontos pela distância dos pontos distribuídos aleatoriamente. Caso o índice encontrado for menor que um, o padrão apresenta aglomeração. Se o índice for maior que um, o padrão tende à dispersão.

\section{Função K de Ripley}

Essa função foi implementada por Ripley (1976) e tem por objetivo a comparação entre uma estimativa empírica e uma resultante de um padrão de pontos espaciais aleatórios (CÂMARA \& CARVALHO, 2004). A comparação demonstra se existe um agrupamento ou dispersão dos eventos dentro de uma quantidade de distâncias pré-escolhidas. Neste estudo, a função K foi utilizada por meio da ferramenta Multi-Distance Spatial Cluster Analysis (MSCA) do programa ArcGIS, que é apresentada na sua forma transformada L(d) na Equação 3 (ESRI, 2015).

$L(d)=\sqrt{\frac{A \sum_{i=1}^{N} \sum_{j=1, j \neq i}^{N} k(i, j)}{\pi N(N-1)}}$

Em que:

$\mathrm{A}=$ área;

$\mathrm{N}$ = número de eventos;

$\mathrm{d}=$ raio de pesquisa;

$k(i, j)=$ peso.

Para verificar o agrupamento ou dispersão entre as ocorrências, foram utilizados três RPs: (d) 100, 150 e 200 m. Com d, determinaram-se as intensidades N/A dos valores observados e esperados em envelopes de confiança (ECs). Além de "d", para aplicação da ferramenta MSCA fixaram-se os seguintes parâmetros:

- nove faixas de distâncias, que visam modificar o número de vezes em que serão aumentadas as vizinhanças para análise do padrão de eventos;

- 99 ECs, onde são colocados pontos de forma aleatória na área de estudo com a finalidade de criar os ECs; e

- $50 \mathrm{~m}$ de incremento de distância, sendo a distância que será incrementada para análise a cada vez que a vizinhança obtiver um aumento.

Para os demais parâmetros, consideraram-se as configurações padrões do programa. Mais esclarecimentos sobre a aplicação da função K de Ripley, com o programa ArcGIS, podem ser consultadas no trabalho de Scott e Janikas (2010).
O uso da função transformada facilita a interpretação gráfica da função K de Ripley (PEREIRA et al., 2013), na qual valores positivos de $\mathrm{L}(\mathrm{d})$ representam que há agrupamento na escala considerada. Dessa forma, se todos os valores de $\mathrm{L}(\mathrm{d})$ positivos ficarem acima do EC, o agrupamento está em toda escala de estudo. Já os valores de L(d) negativos e abaixo do EC confirmam a repulsão entre as ocorrências analisadas. Para os valores dentro do EC, são considerados aleatórios.

Em outras palavras, o resultado dessa função tem, para um conjunto aleatório de pontos, a mesma distância (d) de entrada e é demonstrado na forma de um gráfico que contém os valores observados de K (VOK), os valores esperados de K (VEK) e os ECs. Quando o VOK é maior que o VEK, para determinada distância, os eventos são considerados agrupados. Do contrário, diz-se que os eventos são dispersos. O EC serve para mostrar se esse agrupamento ou dispersão é estatisticamente significante, isto é, quando o VOK for superior ao valor do EC superior, o agrupamento é dito estatisticamente significante. O contrário também é válido; portanto, caso o VOK seja menor que o valor do EC inferior, a dispersão é dita estatisticamente significante.

\section{RESULTADOS E DISCUSSÃO}

\section{Análises qualitativas da água}

A Tabela 1 apresenta os resultados das análises de qualidade da água em cada ponto de monitoramento. A partir dos resultados, observa-se melhor qualidade no P1, CORSAN, localizado na parte alta da bacia. À medida que os rios entram na área urbana, observa-se degradação ambiental acentuada no P2, Vila Brasília (destacando-se elevados valores de DBO e coliformes termotolerantes). A partir do P2, o rio autodepura-se, apresentando uma recuperação ambiental no P3, Monumento, em termos de OD, DBO e coliformes.

Com relação aos gêneros de bactérias, detectou-se a presença de várias, dentre elas algumas da família Enterobacteriaceae, como Klebsiella, Serratia e Enterobacter, que são patógenos oportunistas, e ainda a presença de Aeromonas. Uma parte das bactérias encontradas é bacilo gram-negativo manchada, de hastes não esporuladas, que está associada com as fezes de animais homeotérmicos e com o solo (PELCZAR \& CHAN, 2005).

As bactérias citadas são causadoras de infecções intestinais e/ou do trato urinário. A Escherichia coli é clinicamente a mais importante, uma vez que é um organismo exclusivamente de intestino grosso. Sua presença no intestino contribui para o seu normal funcionamento, no entanto sua presença na água é um indicador de contaminação fecal (JAWETZ \& LEVINSON, 2001).

Três dos cinco gêneros de bactérias foram encontrados somente a partir do ponto de monitoramento da Vila Brasília (P2). Até o P2 ocorreu toda a contribuição urbana referente aos esgotos sanitários, e 
no P3, mesmo estando após uma área de vegetação nativa, as bactérias patogênicas se mantiveram presentes.

As bactérias Klebsiella oxytoca e do gênero Enterobacter, além de serem encontradas no trato intestinal do homem e de outros animais, estão presentes em outros ambientes (JAWETZ \& LEVINSON, 2001). A detecção da Klebsiella oxytoca indica contaminação por despejos de esgotos sanitários. Serratia plimuthyca também é encontrada em ambientes como o solo e a água. E, por fim, nas análises realizadas,

Tabela 1 - Análise qualitativa nos pontos de monitoramento.

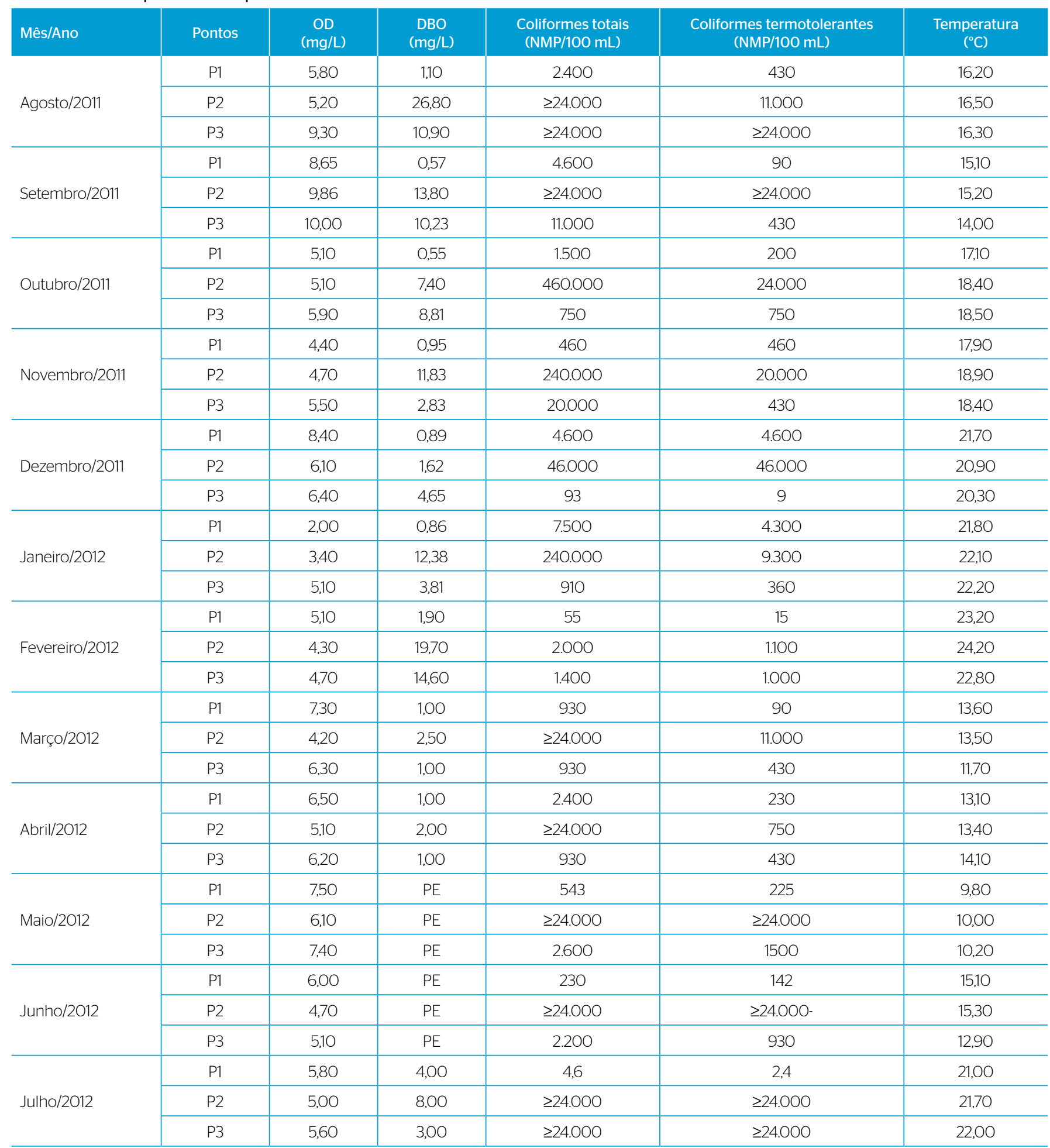

OD: oxigênio dissolvido; DBO: demanda bioquímica de oxigênio; P1: CORSAN; P2: Vila Brasília; P3: Monumento; PE: Problema no equipamento. 
encontraram-se Aeromonas, que são microrganismos de circulação ambiental e com capacidade de ocasionar patogenias moderadas ou graves, tanto no homem quanto em outros animais (RODRIGUES, 2007).

\section{Serviço de esgotamento sanitário e doenças diarreicas no município}

O município de Silveira Martins não possui estação de tratamento de efluentes (ETE), ou seja, os efluentes sanitários são despejados sem tratamento ou possuem sistemas individuais de tratamento (tanques sépticos). No entanto, apenas $16,98 \%$ dos proprietários fazem a manutenção de forma correta desses tanques, e 53,77\% não realizam a limpeza deles (MORAES; SANTOS; FORGIARINI, 2013).

Silveira Martins é um típico município brasileiro de pequeno porte, com menos de 5 mil habitantes. Esses municípios correspondem a um quarto do número total de cidades brasileiras (IBGE, 2010). O potencial de poluição dessas localidades é significativo, a partir da perspectiva de crescimento e a proximidade às áreas rurais preservadas. Além disso, as Companhias Estaduais de Saneamento Básico (CESBs) dificilmente fazem grandes investimentos nesses municípios, devido ao pequeno (ou inexistente) ganho de escala.

No período de realização do estudo, foram observados 34 casos de DDA. Usando o endereço encontrado nas planilhas de registros dos casos de diarreia, foi possível identificar a distribuição espacial dos casos de DDA dentro da área urbana do município (Figura 2).

Observa-se o elevado número de casos de diarreia na Zona Especial de Interesse Social (ZEIS) (20/34 casos - 58,82\%). Esta região está localizada próxima ao segundo ponto de monitoramento (Vila Brasília); nela, as moradias foram construídas às margens do rio. Esse resultado corrobora a associação clássica de que as piores condições de vida estão relacionadas com o maior número de casos de diarreia (TORRES et al., 2013).

As condições ambientais nessa área são preocupantes, visto que há o contato direto da população com a água contaminada, principalmente crianças. Analisando os casos, constatou-se que 95,50\% das ocorrências de DDA são em crianças, e na faixa etária de até 5 anos o percentual é de $89,50 \%$. Queiroz, Heller e Silva (2009) enfatizam que as crianças na faixa etária compreendida entre um e quatro anos são as que mais sofrem com as doenças diarreicas. Constatou-se também que as atividades de lazer das crianças ocorrem junto aos rios, como pode ser observado na Figura 3. Esta figura exibe uma das coletas de água em que as crianças que residem na região foram observadas próximas ao rio poluído, uma situação rotineira. As famílias argumentam que a cidade não tem opções de áreas de lazer adequadas para as crianças. $\mathrm{Na}$ área, não são exercidos os usos de irrigação e dessedentação animal.

$\mathrm{O}$ abastecimento do município é realizado pela CORSAN, que afere a qualidade da água com a frequência estabelecida pela Portaria $\mathrm{n}^{\circ} 518$ do Ministério da Saúde. O atendimento pela rede da CORSAN no município é de 53\%, mas considerando apenas a área urbana, este

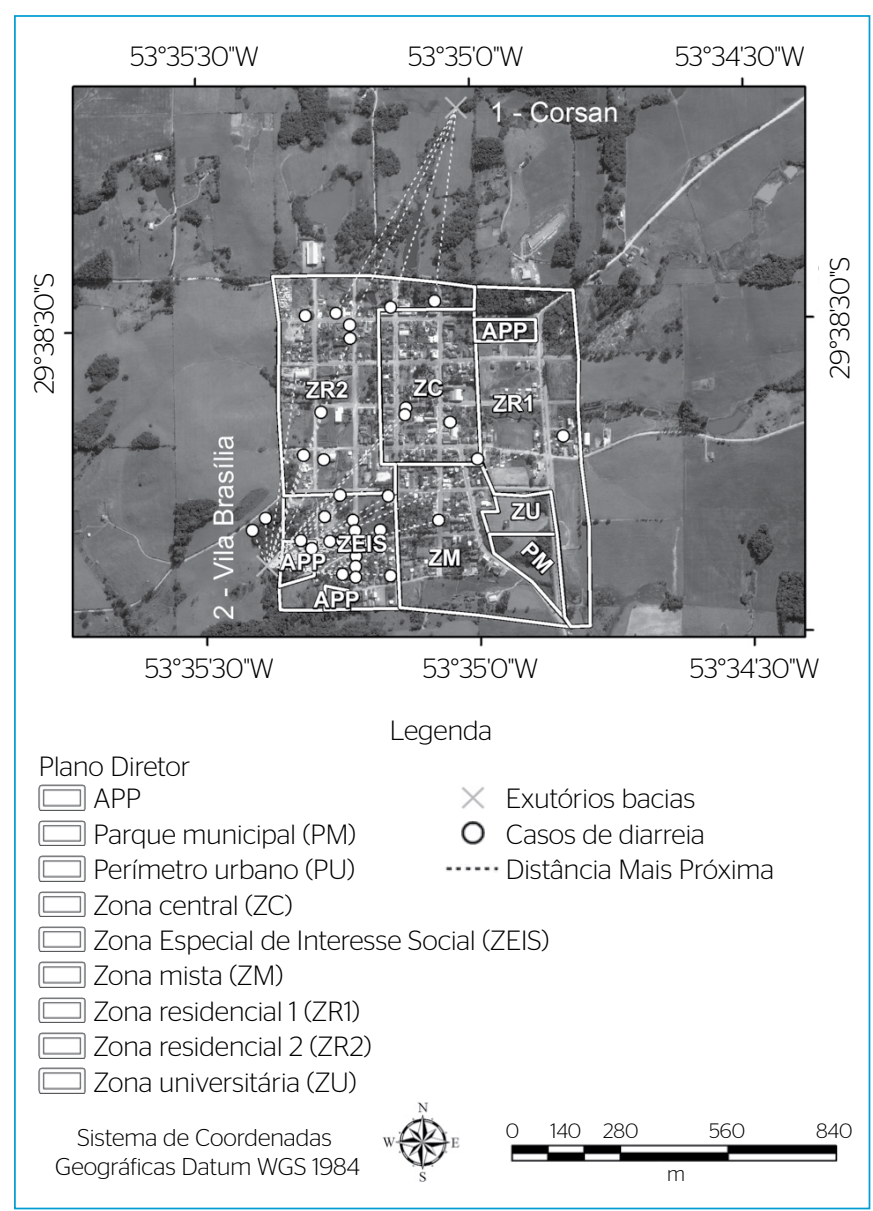

Figura 2 - Casos de diarreia e suas relações com as Zonas do Plano Diretor Municipal e os pontos de monitoramento.

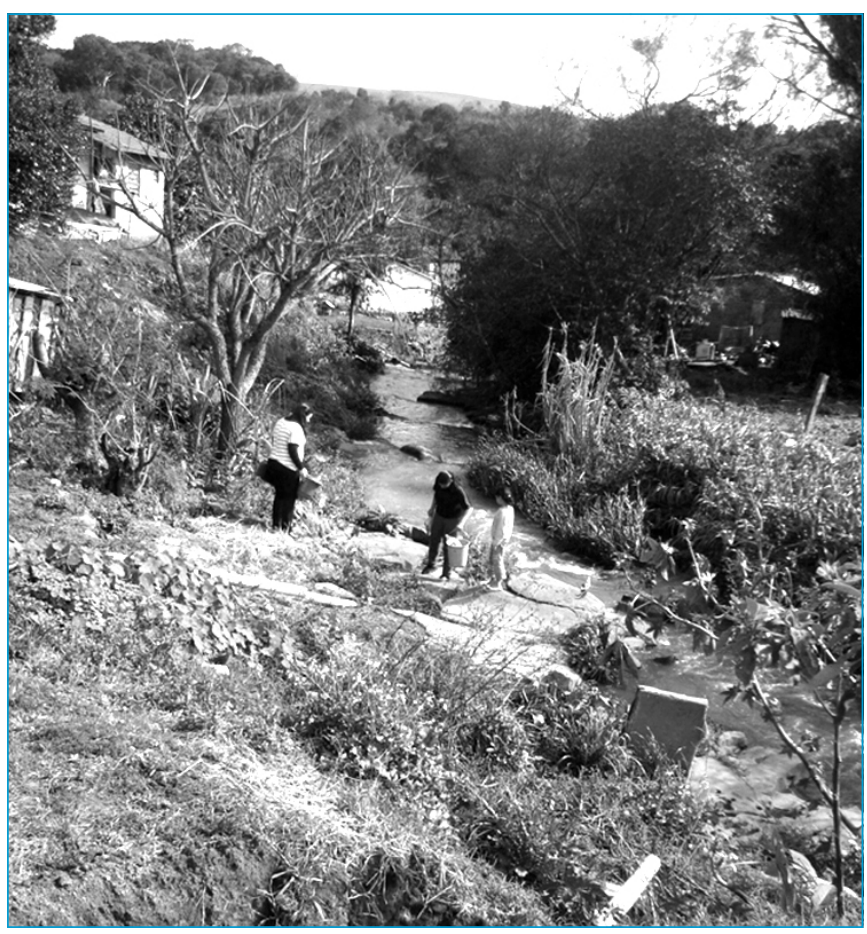

Figura 3 - Condições ambientais e criança brincando próximo ao rio na Vila Brasilia. 
percentual sobe para 84\% (SILVEIRA MARTINS, 2014), valor considerado bom. Sendo assim, as condições sanitárias observadas no município e refletidas nos parâmetros de qualidade da água monitorados nos rios se configuram como um fator relevante para o surgimento de doenças de veiculação hídrica, especialmente as diarreicas. As doenças diarreicas podem ser causa de várias enfermidades (QUEIROZ; HELLER; SILVA, 2009), porém, sabe-se, há algumas décadas, que o investimento em tratamento de efluentes sanitários tem consequências positivas na redução delas (HELLER, 1998).

\section{Análises espaciais}

\section{Análises de distribuição espacial}

A análise espacial de distância mais próxima dos casos de DDA aos pontos de monitoramento mostrou que 4 dos 34 casos são ligados ao P1, CORSAN (Figura 2 - linhas tracejadas que ligam os casos de diarreia aos pontos de monitoramento). No P2 (Vila Brasília), a análise mostrou que todos os outros 30 casos são ligados a ele. Nota-se um padrão entre os casos de DDA e o ponto que recebe a maior carga das águas contaminadas pela disposição irregular de esgotos sanitários na zona urbana do município.

Após a identificação de quais casos seriam ligados aos pontos de monitoramento, foram elaborados os mapas de densidade kernel. As áreas quentes determinadas com diferentes valores de TC não têm um grande impacto na análise e na predição de padrões espaciais (CHAINEY, 2013). Quanto maior for o TC, menor será sua suavização e igualmente menor o tempo de processamento da estimativa kernel. O oposto também é válido, isto é, quanto menor for o TC, maior será sua suavização e exigirá um tempo maior de processamento. Por essa razão, na análise realizada sobre a EDK, foram fixados os valores de TC em 2,5 m utilizando o Método Geodésico e selecionando diferentes parâmetros de RP para, posteriormente, comparar seus resultados.

Seguindo a metodologia proposta por Chainey, Tompson e Uhlig (2008), para a elaboração dos mapas EDK foram utilizadas em sua classificação cinco classes temáticas usando o método Quantile com os valores padrões do ArcGIS para cada RP. A área de estudo foi considerada o retângulo envolvente da camada dos casos de diarreia $\left(744.763 \mathrm{~m}^{2}\right)$; e a área quente, a classe mais intensa, chamada de "muito forte" (Figura 4).

Os RPs de 100, 150 e 200 m geraram áreas quentes de 91,69, 133,39 e $144,87 \mathrm{~m}^{2}$, respectivamente. Os casos de diarreia nas áreas quentes foram de 25 para os raios de 100 e $150 \mathrm{~m}$ e de 22 para o raio de $200 \mathrm{~m}$. Assim, o PAI, para os raios de 100, 150 e 200 m, foi de 6,00, 4,09 e 3,34 , respectivamente.

Os valores de PAI encontrados mostram que o RP que gera a melhor demonstração de áreas quentes é o de $100 \mathrm{~m}$, pois tem valor de PAI mais elevado quando comparado aos outros RPs. Esse resultado indica que a taxa de acerto do número de casos de diarreia é maior na menor distância, ou seja, que o acerto está agrupado no menor raio, estando concentrado na Vila Brasília e próximo ao trecho de rio com pior qualidade da água.

A metodologia promove a suavização estatística dos dados, filtrando as diferenças e retendo as propriedades essenciais (ARAÚJO et al., 2007). Ela traz como resultado uma superfície cujo valor é proporcional à intensidade de amostras por unidade de área. Isso evidencia que a falta de tratamento dos efluentes da cidade de Silveira Martins tem um papel fundamental no aumento da incidência de casos de

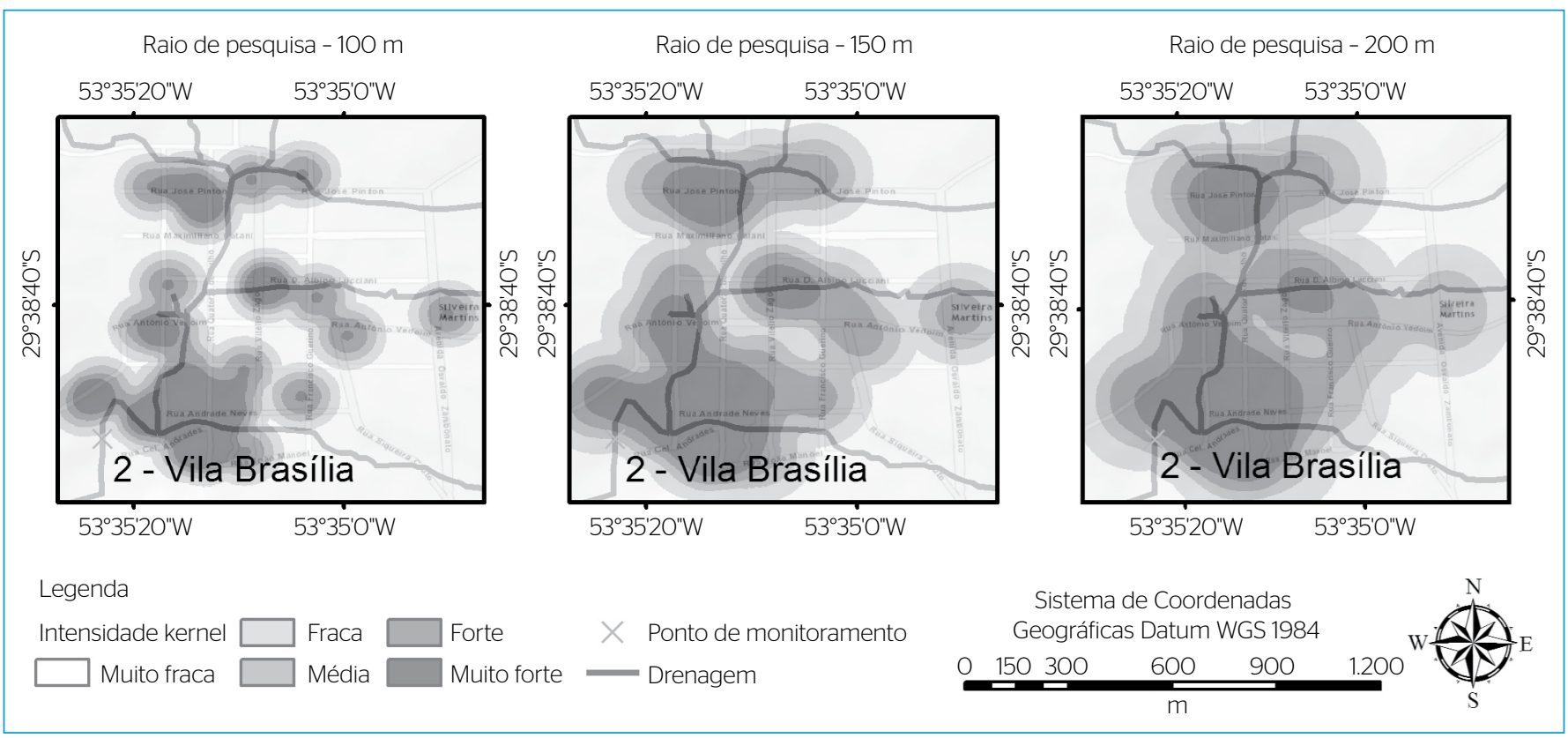

Figura 4 - Mapas de densidade kernel de acordo com os diferentes raios de pesquisa. 
diarreia, pois se trata de uma região onde os moradores estão mais vulneráveis, especialmente as crianças. Chainey (2013) relata que a EDK é uma das técnicas mais precisas para identificar o risco de ocorrência, e a ferramenta é amplamente utilizada em anos recentes na área de saúde e ambiente.

\section{Análises de aglomerados}

Primeiramente, foi usada a função VMP do programa ArcGIS. Com sua área de estudo sendo o menor retângulo envolvente dos casos de diarreia, e usando o método euclidiano para o cálculo das distâncias, foi obtido um valor do índice da VMP de 1,009429 — valor inconclusivo, uma vez que é muito próximo de um.

$\mathrm{Na}$ análise de agrupamento usando a ferramenta baseada na função K de Ripley foi empregada uma distância inicial no mesmo valor do RP da EDK, isto é, $100 \mathrm{~m}$. Para o incremento de distância, foi utilizado valor de $50 \mathrm{~m}$ com 9 faixas de distância e um EC de 99 permutações que resulta em um nível de confiança de 99\%. Com esses valores, foi obtida a Figura 5.

Essa figura demonstra que, para uma distância de até 200 m, existe uma aglomeração estatisticamente significante dos eventos em estudo, pois o VOK é superior tanto ao VEK quanto ao EC superior. Para a distância de 200 a 300 m existe também certo nível de agrupamento, entretanto não é possível afirmar a sua significância estatística, já que o VOK é superior ao VEK, mas não ao EC superior. A partir do valor de $300 \mathrm{~m}$, os eventos começam a ser considerados dispersos devido ao maior VEK em relação ao VOK. Porém, até a distância máxima de $500 \mathrm{~m}$ demonstrada no gráfico, não podemos afirmar que os eventos são estatisticamente significantes para a dispersão.

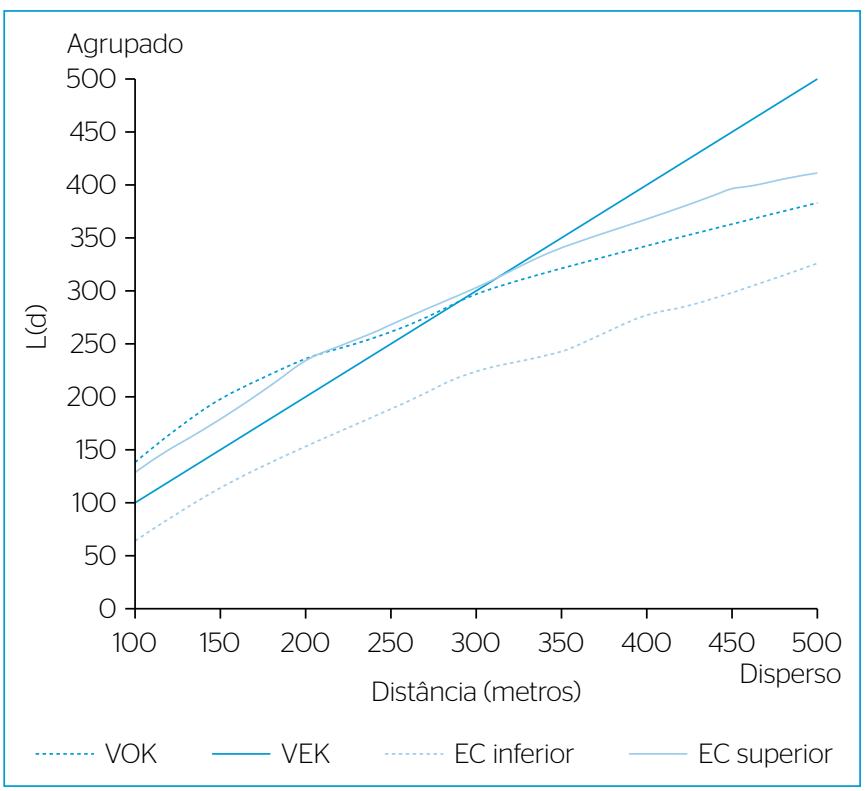

Figura 5 - Análise de agrupamento dos casos de doenças diarreicas agudas usando a função K de Ripley.
Esse resultado confirma os anteriores encontrados na metodologia EDK, isto é, existe uma aglomeração estatisticamente significativa em menores distâncias, demonstrando um agrupamento dos casos de diarreia. Esse agrupamento se deu próximo aos rios que passam pela área urbana do município, especialmente junto ao ponto de monitoramento 2, Vila Brasília (Figura 4).

Na região da Vila Brasília, constataram-se esgotos escoando na via pública, disposição de resíduos sólidos de forma inadequada, inundações dos lotes que ocupam a APP dos rios e crianças brincando nessa área. Todos esses fatores são causas potenciais para doenças diarreicas em crianças de até cinco anos (HELLER, 1998; QUEIROZ; HELLER; SILVA, 2009).

Os resultados obtidos com as metodologias espaciais utilizadas demonstram a necessidade de trabalhar com ações diferenciadas dentro de um mesmo município. Conforme argumenta Ribeiro (2014), as ferramentas de geoprocessamento ajudam a entender a distribuição espacial dos riscos à saúde e a se fazer hipóteses etiológicas, além de enfrentá-los de forma mais eficiente no território, pois permitem identificar as desigualdades e as iniquidades espaciais em saúde. O risco de contrair uma doença de veiculação hídrica está associado à vulnerabilidade (função das características socioeconômicas da população, especialmente) e à ameaça (poluição hídrica que contamina as águas).

A utilização do bairro (TORRES et al., 2013) (ou até mesmo de Zonas do Plano Diretor) como unidade de análise espacial da ocorrência de doenças diarreicas permite uma maior aproximação à realidade local. Além disso, as informações acerca da qualidade dos rios possibilitam identificar a possível relação entre causa e efeito das doenças de veiculação hídrica. Cargas orgânicas e bactérias presentes nos rios potencializam a proliferação de doenças, por meio do contato direto com esse rio ou pela contaminação dos mananciais utilizados para abastecimento. Conforme constataram Beale et al. (2008), é preciso complementar os sistemas de monitoramento de modo a alimentar os modelos de estatística geoespacial.

\section{CONCLUSÕES}

O estudo identificou espacialmente que as doenças diarreicas têm uma área de risco diretamente relacionada com a qualidade dos recursos hídricos (ameaça) e as características socioeconômicas da população (vulnerabilidade). Os casos de diarreia se aglomeram em uma região de menor poder aquisitivo, com residências nas margens do rio que atravessa o município. Nessa região, foram observados os piores resultados das análises de qualidade da água. As análises espaciais de primeira ordem mostram que a intensidade dos casos de diarreia é visualmente mais intensa nas redondezas dessa área urbana.

Nas análises de segunda ordem, a função K de Ripley demonstrou estatisticamente que para um RP de até $200 \mathrm{~m}$, especialmente entre 
100 e 150 m, existem aglomerados nos casos de diarreia. As análises de EDK e a função $\mathrm{K}$ indicam que a poluição que a cidade produz no corpo hídrico é diretamente relacionada com a ocorrência de doenças diarreicas. Assim, os resultados demonstram a importância de se trabalhar com análises espaciais e relacionar os casos observados com o monitoramento ambiental em rios.
Essa realidade é a mesma de diversos municípios brasileiros. As metodologias de análises espaciais descritas podem ser replicadas em outros municípios com objetivo de identificar áreas de risco às doenças de veiculação hídrica. Por meio dessa identificação será possível aplicar ações educativas de prevenção dessas doenças, proporcionando a redução dos casos de internação e a consequente otimização do gasto dos recursos públicos.

\section{REFERÊNCIAS}

AMARAL, L.A. do; NADER FILHO, A.; ROSSI JUNIOR, O.D.; FERREIRA, F.L.A.; BARROS, L.S.S. (2003) Água de consumo humano como fator de risco à saúde em propriedades rurais. Revista de Saúde Pública, v. 37, n. 4, p. 510-514. http://dx.doi.org/101590/S003489102003000400017

AMERICAN PUBLIC HEALTH ASSOCIATION (APHA). (2012) Standard Methods For The Examination of Water and Wastewater. 22. ed. Washington, D.C.: APHA.

ARAÚJO, K.; RESENDES, A.P.C:; SOUZA-SANTOS, R.; SILVEIRA JÚNIOR, J.C.; BARBOSA, C.S. (2007) Análise espacial dos focos de Biomphalaria glabrata e de casos humanos de esquistossomose mansônica em Porto de Galinhas, Pernambuco, Brasil, no ano 2000. Cadernos de Saúde Pública, v. 23, n. 2, p. 409-417. http:// dx.doi.org/10.1590/S0102-311X2007000200017

BEALE, L.; ABELLAN, J.J.; HODGSON, S.; JARUP, L. (2008) Methodologic Issues and Approaches to Spatial Epidemiology. Environmental Health Perspectives, v. 116, n. 8, p. 1105-1110. https:// dx.doi.org/10.1289\%2Fehp.10816

CÂMARA, G.; CARVALHO, M.S. (2004) Análise espacial de dados geográficos. Planaltina: Empresa Brasileira de Pesquisa Agropecuária Cerrados (Embrapa Cerrados). p. 53-122.

CÂMARA, G.; MONTEIRO, A.M.V.; FUCKS, S.D.; CARVALHO, M.S. (2004) Spatial analysis and GIS: a primer. Disponível em: <https:// www.researchgate.net/publication/2934461_Spatial_Analysis_and_ GIS_A_Primer>. Acesso em: 20 jan. 2016.

CARVALHO, M.S.; SOUZA-SANTOS, R. (2005) Análise de dados espaciais em saúde pública: métodos, problemas, perspectivas Analysis of spatial data in public health: methods, problems, and perspectives. Cadernos de Saúde Pública, v. 21, n. 2, p. 361-378. http://dx.doi.org/10.1590/S0102-311X2005000200003

CHAINEY, S.; TOMPSON, L.; UHLIG, S. (2008) The Utility of Hotspot Mapping for Predicting Spatial Patterns of Crime. Security Journal, v. 21, n. $1-2$, p. $4-28$.

CHAINEY, S.P. (2013) Examining the influence of cell size and bandwidth size on kernel density estimation crime hotspot maps for predicting spatial patterns of crime. Bulletin of the Geographical Society of Liege, v. 60, p. 7-19.
DIGGLE, P.J.; CHETWYND, A.G. (1991) Second-Order Analysis of Spatial Clustering for Inhomogeneous Populations. Biometrics, v. 47, n. 3, p. 1155-1163. http://doi.org/10.2307/2532668

DRUCK, S.; CARVALHO, M.S.; CÂMARA, G.; MONTEIRO, A.V.M. (2004) Análise espacial de dados geográficos. Brasil: Embrapa Cerrados.

ENVIRONMENTAL SYSTEMS RESEARCH INSTITUTE (ESRI). (2O15) ARCGIS 10.3.1. Redlands: ESRI.

GATRELL, A.C.; BAILEY, T.C.; DIGGLE, P.J.; ROWLINGSON, B.S. (1996) Spatial Point Pattern Analysis and Its Application in Geographical Epidemiology. Transactions of the Institute of British Geographers, v. 21, n. 1, p. 256-274. http://doi.org/10.2307/622936

HELLER, L. (1998) Relação entre saúde e saneamento na perspectiva do desenvolvimento. Ciência \& Saúde Coletiva, Rio de Janeiro, v. 3 , n. 2, p. 73-84. http://dx.doi.org/10.1590/S1413-81231998000200007

INSTITUTO BRASILEIRO DE GEOGRAFIA EESTATÍSTICA (IBGE). (2010) Censo 2010: primeiros resultados. Disponível em: <http://www.bge. gov.br/home/estatistica/populacao/censo2010/primeiros_resultados/ default_primeiros_resultados.shtm>. Acesso em: 8 ago. 2011.

JAWETZ, E.; LEVINSON, W. (2001) Microbiologia médica e imunologia. São Paulo: Artmed.

MORAES, L.M.;SANTOS, T.P.; FORGIARINI, F.R. (2O13) Bactérias de origem fecal: análise espacial e sugestões para a melhoria da saúde pública. In: SIMPÓSIO DE HIDRÁULICA E RECURSOS HIIDRICOS DOS PAÍSES DE LÍNGUA PORTUGUESA, 11., 2013, Maputo, Moçambique. Anais...

PELCZAR, M.J.; CHAN, E.C.S. (2005) Microbiologia: conceitos e aplicações. São Paulo: Pearson Makron Books.

PEREIRA, A.A.; BARROS, D.A. de; ACERBI JUNIOR, F.W.; PEREIRA, J.A.A.; REIS, A.A. dos. (2013) Análise da distribuição espacial de áreas queimadas através da função K de Ripley. Scientia Forestalis, Piracicaba, v. 41, n. 100, p. 445-455.

QUEIROZ, J.T.M.; HELLER, L.; SILVA, S.R. (2009) Análise da correlação de ocorrência da doença diarreica aguda com a qualidade da água para consumo humano no município de Vitória-ES. Saúde e Sociedade, São Paulo, v. 18, n. 3, p. 479-489. http://dx.doi.org/10.1590/ S0104-12902009000300012 
RIBEIRO, H. (2014) Geografia da saúde no cruzamento de saberes. Saúde e Sociedade, São Paulo, v. 23, n. 4, p. 1123-1124. https://doi. org/10.1590/S0104-12902014000400200

RIPLEY, B.D. (1976) The Second-Order Analysis of Stationary Point Processes. Journal of Applied Probability, v. 13, n. 2, p. 255-266. https://doi.org/10.2307/3212829

RODRIGUES, E. (2007) Pesquisa de Aeromonas spp. em tilápia (Oreochromis niloticus), cultivada no estado do Rio de Janeiro-Brasil: isolamento, identificação de espécies e avaliação da sensibilidade antimicrobiana. Tese (Doutorado)-Universidade Federal Fluminense, Rio de Janeiro.

SCOTT, L.M.; JANIKAS, M.V. (2010) Spatial Statistics in ArcGIS. In: FISCHER, M.M.; GETIS, A. (Orgs.). Handbook of Applied Spatial Analysis. Berlim, Heidelberg: Springer Berlin Heidelberg. p. 27-41.

SILVEIRA MARTINS. (2014) Plano Municipal de Saneamento Básico - Silveira Martins/RS. Silveira Martins. Disponível em: <http:// silveiramartins.rs.gov.br/wp-content/uploads/2010/O7/PLANODE-SANEAMENTO-PMSM-VERS\%C3\%830-FINAL-jan-2014-2.pdf>. Acesso em: 15 jul. 2016.

SILVERMAN, B.W. (1986) Density estimation for statistics and data analysis. Flórida: CRC Press. v. 26.
SIQUEIRA-JUNIOR, J.B.; MACIEL, I.J.; BARCELLOS, C.; SOUZA, W.V.; CARVALHO, M.S.; NASCIMENTO, N.E.; OLIVEIRA, R.M.; MORAIS-NETO, O.; MARTELLI, C.M.T. (2008) Spatial point analysis based on dengue surveys at household level in central Brazil. BMC Public Health, v. 8, n. 1, p. 361. https://doi. org/10.1186/1471-2458-8-361

TORRES, R.M.C.; BITTENCOURT, S.A.; OLIVEIRA, R.M.; SIQUEIRA, A.S.P.; SABROZA, P.C.; TOLEDO, L.M. (2013) Use of local level indicators for the spatial analysis of morbidity due to diarrhea and its relation with lifestyle conditions. Ciência \& Saúde Coletiva, v. 18, n. 5, p. 1441-1450. http://dx.doi.org/10.1590/S141381232013000500029

VIANA, R.L.; FREITAS, C.M. de; GIATTI, L.L. (2016) Saúde ambiental e desenvolvimento na Amazônia legal: indicadores socioeconômicos, ambientais e sanitários, desafios e perspectivas. Saúde e Sociedade, São Paulo, v. 25, n. 1, p. 233-246. http://dx.doi. org/10.1590/S0104-12902016140843

VILAÇA, P.J.; ALBUQUERQUE, J.O.M.; SANTOS, M.G.S.; GLASSER, R.G.; GENEHR, L.; SANTANA, C.A.; BANCHER, A.M. (2009) Identificação e Delimitação de Áreas Prioritárias para Controle da Leptospirose no Município de São Paulo. Saúde e Sociedade, São Paulo, v. 18, supl. 1, p. 82-83. http://dx.doi.org/10.1590/S010412902009000500039 